\title{
Improved outcomes in metastatic germ cell cancer: results from a large cohort study
}

\author{
Marcus Hentrich $^{1}\left[\right.$ ] Jessica Debole ${ }^{2,3} \cdot$ Vindi Jurinovic $^{4} \cdot$ Arthur Gerl $^{2}$
}

Received: 30 April 2020 / Accepted: 30 July 2020 / Published online: 9 August 2020

(c) The Author(s) 2020

\begin{abstract}
Purpose Treatment of metastatic germ cell cancer (GCC) is based on the International Germ Cell Cancer Collaborative Group (IGCCCG) prognostic classification published in 1997. 5-year survival rates were reported to be $91 \%, 79 \%$, and $48 \%$ for patients with good, intermediate and poor prognosis, respectively. However, treatment results may have improved over time due to cumulative experience, improved supportive care and modern-type chemotherapy.

Methods Patients with metastatic GCC who received cisplatin-based chemotherapy at two institutions in Munich between 2000 and 2013 were retrospectively studied. Clinical characteristics, treatment and outcomes were analyzed with respect to the IGCCG prognostic classification.

Results Of 225 patients (median age 35 years), 72 (32\%) had seminoma (S) and 153 (68\%) nonseminoma. 175 (78\%), 30 (13\%) and 20 patients $(9 \%)$ had good, intermediate and poor prognosis according to the IGCCCG classification. The 2-yearprogression free survival of patients with good, intermediate and poor prognosis was $91 \%, 83 \%$ and $37 \%$, and the 5-yearoverall survival (OS) was $98 \%, 96 \%$, and $66 \%$, respectively. There was no significant difference in the OS between patients in the good and intermediate prognosis group.

Conclusion Compared to data from the original IGCCCG classification system, the outcome of patients with metastatic GCC has considerably improved over time. While the prognosis of intermediate-risk patients is excellent, treatment in the poor-prognosis group remains to be improved.
\end{abstract}

Keywords Germ cell cancer · Germ cell tumors · Seminoma · Non-seminoma · IGCCCG classification

This work was presented in part at the 41st Congress of the European Society for Medical Oncology, Copenhagen, Denmark, October 7-11, 2016, and at the Annual Meeting of the German, Austrian and Swiss Societies of Hematology and Medical Oncology, Leipzig, Germany, October 14-18, 2016.

Marcus Hentrich

marcus.hentrich@swmbrk.de

1 Department of Hematology and Oncology, Red Cross Hospital Munich, Ludwig-Maximilians-University Munich, Nymphenburger Str. 163, 80634 Munich, Germany

2 Private Practice of Oncology, Munich, Germany

3 Helios Klinikum München West, Munich, Germany

4 Institute of Biometrics and Epidemiology, Ludwig-Maximilians-University Munich, Munich, Germany

\section{Introduction}

Testicular germ cell cancer is the most common cancer in men aged 18-40 years with an estimated 4120 new cases detected in Germany in 2016 (Robert-Koch-Institute 2019). Today, a cure is expected in $95 \%$ of all patients diagnosed with testicular cancer and in approximately $80 \%$ of patients with metastatic disease (Hanna and Einhorn 2014). Treatment of metastatic germ cell cancer (GCC) is based on the 1997 International Germ Cell Cancer Collaborative Group (IGCCCG) prognostic classification (IGCCCG 1997; Beyer et al. 2013; Albers et al. 2015; Honecker et al. 2018). Three cycles of cisplatin, etoposide, bleomycin (BEP) combination chemotherapy have become standard of care for goodrisk patients while four cycles of BEP are the reference for intermediate- and poor-risk patients (Beyer et al. 2013; Honecker et al. 2018). According to the IGCCCG cohort, five-year survival rates are $91 \%, 79 \%$, and $48 \%$ for patients with good, intermediate and poor prognosis, respectively 
(IGCCCG 1997). However, the IGCCCG classification is based on treatments applied between 1975 and 1990. Recent data indicate that the outcome of patients with metastatic GCC may have improved over time due to cumulative experience of treating physicians, improved supportive care and modern-type chemotherapy (van Dijk et al. 2006; Raggi et al. 2015). The aim of the present study was to analyze the outcome of patients with metastatic GCC treated between 2000 and 2013 at two experienced institutions in Munich, Germany. We presumed that the outcome of metastatic GCC has considerably improved compared to the original IGCCCG experience.

\section{Methods}

All patients who underwent first line chemotherapy between January 2000 and December 2013 at Private Practice of Oncology and at the Department of Hematology and Oncology, Harlaching Hospital, both located in Munich, were identified through the institutional database. Inclusion criteria were (1) pathology proven metastatic GCC, (2) age $\geq 17$ years, (3) start of chemotherapy for metastatic GCC between January 2000 and December 2013, and (4) at least 2-years follow-up. Patients were excluded if data on tumor stage, treatment or follow-up were incomplete.

The medical records were reviewed with regard to age at time of initial diagnosis, histopathology, stage, tumor marker levels, metastatic spread, type and duration of chemotherapy, and outcome.

The primary endpoint was the 5-years overall survival (OS) rate. Secondary endpoints included progression-free survival (PFS) as well as PFS and OS of relapsed patients. The study was approved by the Ethics committee of LudwigMaximilians-University Munich. The study was performed in accordance with the Declaration of Helsinki.

\section{Statistics}

PFS was measured from the beginning of the chemotherapy to the time of progression, relapse or death. OS was calculated from the beginning of the chemotherapy to last follow-up or to death from any cause. Patients without an event were censored at the date of last follow-up. The probability of PFS and OS was determined by the Kaplan-Meier Method and differences between subgroups of patients were assessed by the log-rank test. Statistical analyses including descriptive statistics such as frequency, mean, median, range, inter-quartile range, minimum and maximum were performed by using SPSS software (IBM SPSS Statistic, version 21.0). All p-values were two-sided. $P$ values of 0.05 or less were considered statistically significant.

\section{Results}

\section{Patient characteristics}

Of 255 patients identified, 30 were excluded due to incomplete data. Main characteristics of the 225 patients included into the study are outlined in Table 1 . The median age of the cohort was 35 years (range 17-66). Seminoma and nonseminoma were diagnosed in $72(32 \%)$ and $153(68 \%)$ patients, and 204 patients $(91 \%)$ had a primary gonadal GCC. The cohort includes 56 patients managed with active surveillance for stage I disease who developed metastases after a median time of 9.5 months (range $0.5-15$ ). According to the IGCCCG classification system, 175 (78\%), 30 (13\%) and 20 patients $(9 \%)$ had good, intermediate and poor prognosis GCC as compared to $60 \%, 26 \%$ and $14 \%$ in the original IGCCCG cohort.

Table 1 Patients characteristics and first line treatment

\begin{tabular}{ll}
\hline Patients $(n)$ & 225 \\
Median age & $35(17-66)$ \\
Pathology & \\
Seminoma & $72(32 \%)$ \\
Non-seminoma & $153(68 \%)$ \\
Primary location & \\
Gonadal & $204(91 \%)$ \\
Retroperitoneal & $18(8 \%)$ \\
$\quad$ Seminoma & 9 \\
$\quad$ Non-seminoma & 9 \\
Mediastinal & 3 \\
$\quad$ Non-seminoma & 3 \\
Metastatic sites & \\
Abdominal & $180(80 \%)$ \\
Lung & $49(22 \%)$ \\
Liver & $9(4 \%)$ \\
Bone & $2(1 \%)$ \\
Brain & $4(2 \%)$ \\
Other & $4(2 \%)$ \\
Relapse from Stage I & $56(25 \%)$ \\
Median time to progression & 9.5 month \\
& $($ range \\
IGCCCG risk at relapse from Stage I & $0.5-15)$ \\
Good & \\
IGCCCGmediate & 52 \\
Good & 4 \\
Intermediate & \\
Poor & $175(78 \%)$ \\
\hline & $30(13 \%)$ \\
Mrognostic group (entire cohort) & $20(9 \%)$ \\
\hline
\end{tabular}

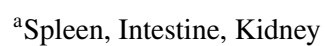


Table 2 First line treatment for metastatic GCC

\begin{tabular}{ll}
\hline $\begin{array}{l}\text { Good prognosis }(n=175) \\
3 \times \text { PEB }^{\mathrm{a}}\end{array}$ & $159(91 \%)$ \\
$4 \times \mathrm{PEB}^{2}$ & $9(5 \%)$ \\
$4 \times \mathrm{PE}$ & $3(2 \%)$ \\
Other & $4(2 \%)$ \\
Intermediate prognosis $(n=30)$ & \\
$4 \times \mathrm{PEB}$ & $25(83 \%)$ \\
$2 \times \mathrm{PEB}->2 \times \mathrm{VIP}$ & $1(3 \%)$ \\
$3 \times \mathrm{PEB}$ & $4(13 \%)$ \\
Poor prognosis $(n=20)$ & \\
$4 \times \mathrm{PEB}$ & $13(65 \%)$ \\
$1 \times \mathrm{VIP}->3 \times \mathrm{PEB}$ & $4(20 \%)$ \\
$\mathrm{VIP}+\mathrm{HDCT}$ & $3(15 \%)$ \\
\hline
\end{tabular}

${ }^{\mathrm{a}}$ Including PEB $\longrightarrow$ VIP $(n=3)$

${ }^{\mathrm{b}} \mathrm{PE} \times 3(n=3), \mathrm{PEB} \times 2(n=1)$

Type of first line chemotherapy and the number of cycles given are shown in Table 2. The vast majority of patients received three to four cycles of platinum-based chemotherapy, while primary high-dose chemotherapy (HDCT) was applied to three patients in the poor prognosis group. All nonseminoma patients with residual tumor $>1 \mathrm{~cm}$ after chemotherapy were routinely scheduled for secondary surgery. Thus, 33 of 225 (15\%) patients underwent secondary retroperitoneal lymph node dissection $(n=28)$, and/or secondary surgery of the lungs $(n=5)$, liver $(n=1)$, mediastinal $(n=3)$ and infraclavicular lymph nodes $(n=1)$. Histopathological examination revealed necrosis/fibrosis in 16 of 38 (42\%) resection specimens, teratoma in $10(26 \%)$, vital GCC in 7 (18\%), and malignancies other than GCC in $5(13 \%)$ (spindle cell tumor $[n=3]$, undifferentiated non-small cell carcinoma [ $n=1]$, not specified $[n=1])$.

Salvage treatment in relapsed patients was applied to 14 , 5 and 11 patients of the good, intermediate and poor prognosis group, respectively. It consisted of surgery alone for teratoma in seven of 30 (23\%) patients, all but one of whom are alive in ongoing remission. Conventional dose first salvage chemotherapy (CDCT) and HDCT were applied to 16 and 7 patients, respectively. CDCT regimens used were cisplatin, etoposide, ifosfamide (VIP, $n=13$ ), cisplatin, ifosfamide, paclitaxel (TIP, $n=1$ ), and gemcitabine, oxaliplatin, paclitaxel (GOP, $n=2$ ). Second and higher relapses occurred in twelve of 30 patients (40\%). Second salvage chemotherapy consisted of GOP $(n=2)$, gemcitabine, paclitaxel $(n=2)$, cisplatin, epirubicine $(n=1)$, oxaliplatin, paclitaxel $(n=1)$, oxaliplatin, irinotecan $(n=1)$, and HDCT $(n=5)$. Of the 12 patients undergoing second salvage chemotherapy, 6 died of GCC while the others are alive and well. Palliative treatment with everolimus was given to two patients within a clinical trial (Fenner et al. 2019).

\section{Survival}

After a median follow-up of 95 months (95\% confidence interval [CI], 88-104), the 2-year-PFS of patients with good, intermediate and poor prognosis was $91 \%$ (95\% CI 87-96), 83\% (95\% CI 70-98) and 37\% (95\% CI 20-66) (Fig. 1). The corresponding 5-year-PFS of the original IGCCCG cohort has been reported to be $88 \%, 78 \%$ and $41 \%$. In our analysis the difference between the good and poor prognosis group $(P<0.0001)$ and between the intermediate and poor prognosis group $(P=0.00045)$ was statistically significant. By contrast, the difference between the good and intermediate group was not statistically significant $(P=0.281)$.

Overall, eleven of 225 (5\%) patients have died, ten of which from GCC and one from community-acquired pneumonia unrelated to cancer treatment (Table 3). The 5-yearOS of our cohort was $98 \%$ (95\% CI 95-100), $96 \%$ (95\% CI 90-100), and 66\% (95\% CI 47-93), for the good, intermediate and poor prognosis group, respectively (Fig. 2; Table 3). This compares favorably to the 5-year-OS of $91 \%, 79 \%$ and $48 \%$ predicted by the original IGCCCG score. The corresponding mean survival times of our cohort were 152 (95\% CI 148-155), 147 (95\% CI 139-156), and 100 months (95\% CI 72-128). There was no significant difference in the OS between patients in the good and intermediate prognosis group $(P=0.754)$. By contrast, the OS of patients from the good and the intermediate prognosis group was significantly superior to that of the poor prognosis group $(P<0.0001$ and $P=0.004$, respectively). Further, the 5-year-OS was significantly better in poor-risk patients without non-pulmonary

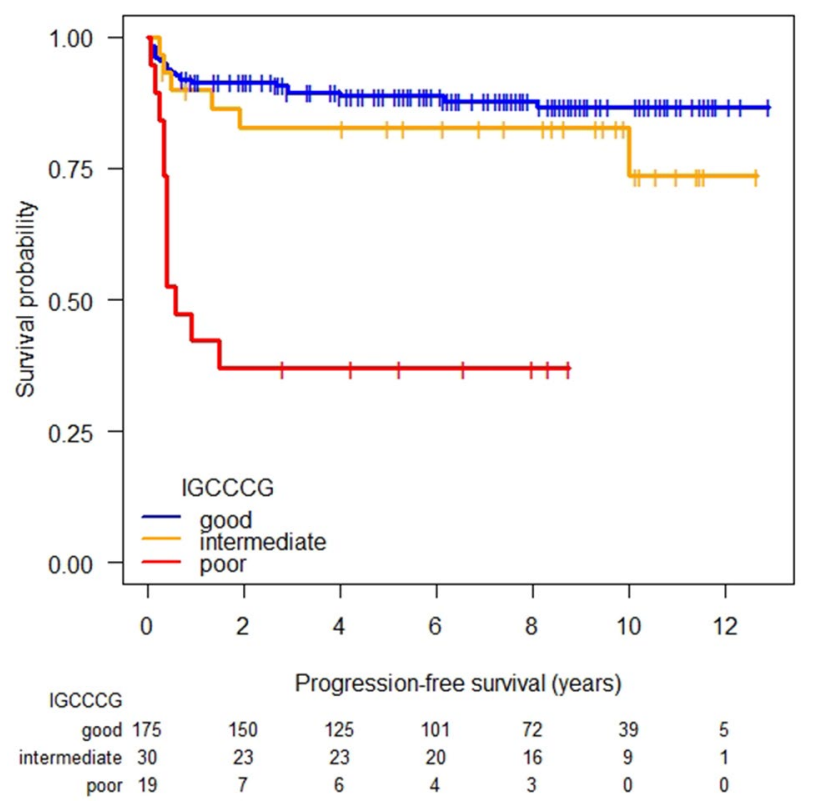

Fig. 1 Progression-free survival according to IGCCCG prognostic groups 
Table 3 Outcome according to the IGCCCG risk classification

\begin{tabular}{ll}
\hline 2-years PFS & \\
Good & $91 \%$ (95\% CI 87-96) \\
Intermediate & $83 \%$ (95\% CI 70-98) \\
Poor & $37 \%(95 \%$ CI 20-66) \\
5-years OS & \\
Good & $98 \%(95 \%$ CI 95-100) \\
Intermediate & $96 \%(95 \%$ CI 90-100) \\
Poor & $66 \%(95 \%$ CI $47-93)$ \\
Relapse/progression & $30 / 225(13 \%)$ \\
Good & $14 / 175(8 \%)$ \\
Intermediate & $5 / 30(17 \%)$ \\
Poor & $11 / 20(55 \%)$ \\
Death & $11 / 225(5 \%)$ \\
Good $(n)$ & $4 / 175(2 \%)$ \\
Intermediate $(n)$ & $1 / 30(3 \%)$ \\
Poor $(n)$ & $6 / 20(30 \%)$ \\
\hline
\end{tabular}

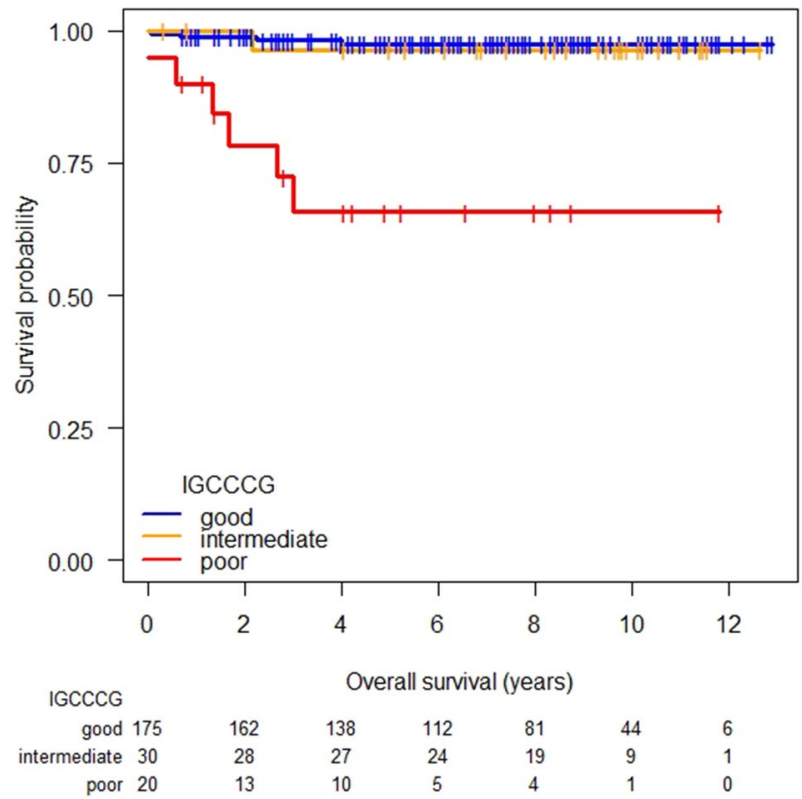

Fig. 2 Overall survival according to IGCCCG prognostic groups

visceral metastasis (NPVM) compared to patients with NPVM (5-year-OS $100 \%$ vs. $38 \%, P=0.033$ ).

The cumulative 5-year-OS of relapsed patients was $75 \%$ (95\% CI 60-93) without significant differences between the good, intermediate and poor prognosis group (Fig. 3) $(P=0.238)$. Of 21 patients treated with first salvage cisplatin-based CDCT $(n=14)$ or carboplatin-based HDCT $(n=7)$ the International Prognostic Factors Study Group (IPFSG) scores were very low $(n=2)$, low $(n=3)$, intermediate $(n=5)$, high $(n=6)$, and very high $(n=5)$, respectively

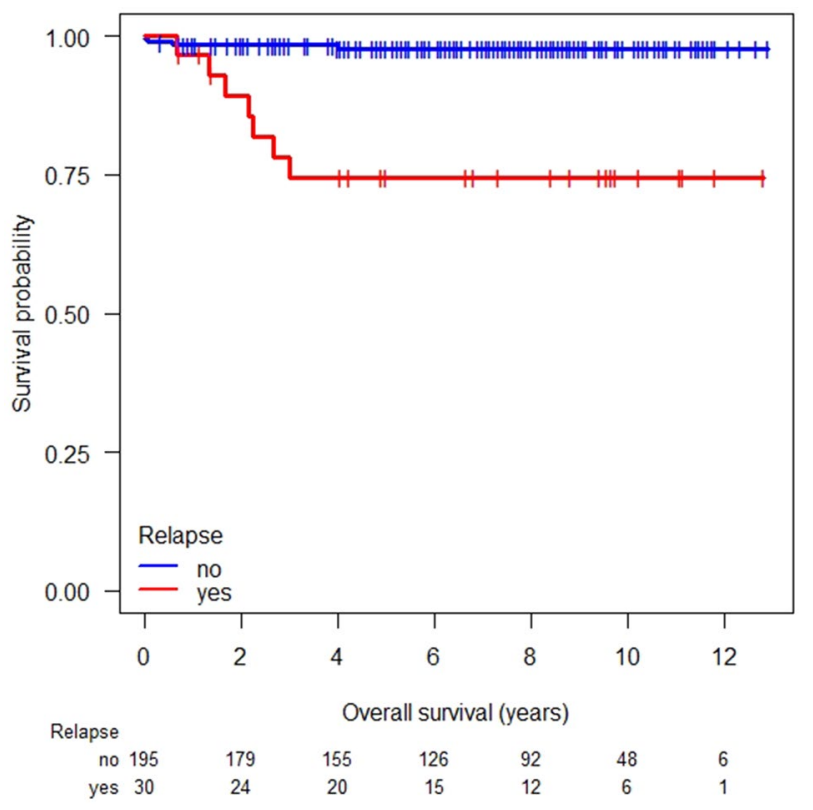

Fig. 3 Overall survival in relapsed vs. non-relapsed patients

(International Prognostic Factors Study Group et al. 2010). However, differences in survival between groups were not calculated due to low number of patients in each group.

\section{Discussion}

There are two major findings from our study. First, the 5-year-OS of patients with metastatic GCC has markedly improved compared to data from the 1997 IGCCCG classification system. The 5-year OS probabilities were 98\% vs. $91 \%, 96 \%$ vs. $79 \%$, and $66 \%$ vs. $48 \%$ for patients in the good-, intermediate- and poor-risk group, respectively. As we did not observe similar improvements in the PFS, the efficacy of first-line chemotherapy in metastatic GCC appears to be unchanged over the last decades. Indeed, while the substitution of etoposide for vinblastine as well as cumulative experience translated into improved survival rates in the early nineties (Williams et al. 1987; Gerl et al. 1996), a number of randomized controlled-trials on first-line chemotherapy published in the last two decades failed to demonstrate significant improvements in survival over standard BEP (Nichols et al. 1998; Motzer et al. 2007; Daugaard et al. 2011; de Wit et al. 2012; Fizazi et al. 2014; Necchi et al. 2015). By contrast, the improvement in the 5-years OS is likely due to salvage therapies that were given more consequently to a higher proportion of patients with relapsed GCC than this may have been the case some decades ago. In fact, standard first salvage regimens such as VIP, TIP or HDCT were applied as first salvage to the vast majority of relapsed patients if they did not undergo curative surgery for 
teratoma only (Kondagunta et al. 2005; Lorch et al. 2012; Adra et al. 2017). Further, adherence to structured follow-up schedules may have contributed to earlier diagnosis of GCC relapses (Albers et al. 2015; Cathomas et al. 2011).

Second, we observed no significant difference in the OS between good-risk and intermediate-risk patients. Thus, the IGCCCG prognostic score no longer predicts different survival probabilities for the groups of good and intermediate-risk patients. Similar data were recently reported from a Swiss cohort that included 204 patients treated from 1991 to 2016 (Fankhauser et al. 2018). As in our study, no significant difference in overall survival between good and intermediate risk patients was found. Another large retrospective observational study on 707 intermediate prognosis GCC patients reported a 5-year-OS rate of $89 \%$ in patients treated from 1997 to 2016, which was significantly superior to the $83 \% 5$-years OS rate of 237 patients treated from 1979 to 1996 (Seidel et al. 2018). Of note, a lactate dehydrogenase (LDH) level $>2$ upper limit of normal (UNL) and AFP levels of $>6200 \mathrm{IU} / \mathrm{ml}$ were independently associated with inferior survival in this study. Further, first-line treatment with three cycles of BEP was given to $53(7 \%)$ patients with no significant differences found in OS and PFS between patients treated with three and four cycles of BEP. However, patient numbers were too low to draw any firm conclusion on whether de-intensification of treatment may be an option. In a subsequent analysis of the cohort intermediate risk patients with AFP values $>6000 \mathrm{IU} / \mathrm{ml}$ and $\mathrm{LDH}>3 \mathrm{UNL}$ revealed an outcome similar to the poor prognosis category (Seidel et al. 2019). Moreover, patients with AFP levels > 1982 IU/ $\mathrm{ml}$ had a higher risk of disease recurrence (recurrence rate $20 \%$ vs. $31 \% ; P=0.02$ ) as did patients with LDH levels $>2$ UNL (recurrence rate $29 \%$ vs. $42.5 \% ; P=0.047$ ). Thus, based on these findings and given the lack of data from prospective randomized trials, three cycles of BEP may only be used on an individual basis in intermediate risk patients with $\mathrm{LDH}$ level $\leq 2 \mathrm{UNL}$ and AFP levels $\leq 1982 \mathrm{IU} / \mathrm{ml}$ (Seidel et al. 2018, 2019). A randomized controlled trial comparing three with four cycles of BEP in intermediate risk patients without high-risk features is warranted.

Our findings compare favorably with recent results from an analysis of a large data set collected to redefine the IGCCCG classification (Gillessen et al. 2019; Beyer et al. 2020). An international consortium contributed data on 9530 advanced non-seminoma GCC patients treated with cisplatin/etoposide based first line chemotherapy between 1990 and 2013. The 5 -years OS was $96 \%, 89 \%$ and $67 \%$ for patients in the good-, intermediate- and poor-risk group, respectively (Gillessen et al. 2019). Further, based on data from 2302 advanced seminoma patients, 5-years OS rates were reported to be $95 \%$ and $87 \%$ for good- and intermediate-risk patients, respectively (Beyer et al. 2020). It should be noted that our data were also included in the IGCCCG project.
The $66 \% 5$-years OS rate of poor-risk patients remains unsatisfactory although this represents an improvement compared to the original IGCCCG risk classification. However, attempts to improve overall survival by intensification of first line chemotherapy have failed (Motzer et al. 2007; Daugaard et al. 2011; Necchi et al. 2015), although a significant PFSadvantage was achieved by use of an intensified chemotherapy schedule for patients with inappropriate tumor marker decline (Fizazi et al. 2014).

Limitations of the present study are its retrospective design and the relatively small sample size. However, our analysis provides important real-life data on clinical outcomes of patients with metastatic GCC mostly managed outside a clinical trial setting.

In conclusion, compared to data from the original IGCCCG classification system, the outcome of patients with metastatic GCC has markedly improved. While the prognosis of intermediate-risk patients is excellent, treatment in the poor-prognosis group remains to be improved.

Acknowledgements Open Access funding provided by Projekt DEAL.

Author contributions MH: study concept and design, data assembly, data analysis and interpretation, writing — original draft, and writingreview and editing. JD: study concept and design, data assembly, data analysis and interpretation, statistical analysis, writing — critical review and editing. VJ: data analysis and interpretation, statistical analysis, writing - critical review and editing. AG: study concept and design, data assembly, data analysis and interpretation, writing-critical review and editing.

Funding This research did not receive any specific grant from funding agencies in the public, commercial, or non-for-profit sectors.

Data availability The data that support the findings of this study are available on request from the corresponding author. The data are not publicly available due to privacy or ethical restrictions.

Code availability Not applicable.

\section{Compliance with ethical standards}

Conflict of interest All authors certify that they have no conflict of interest in the subject matter or materials discussed in this manuscript.

Ethics approval and consent to participate Ethics Committee of Ludwigs-Maximilians-University Munich (UE-No. 009-14) approved the use of these data in 2014 without the need for individual subject consent.

Consent for publication Not applicable.

Open Access This article is licensed under a Creative Commons Attribution 4.0 International License, which permits use, sharing, adaptation, distribution and reproduction in any medium or format, as long as you give appropriate credit to the original author(s) and the source, provide a link to the Creative Commons licence, and indicate if changes were made. The images or other third party material in this article are included in the article's Creative Commons licence, unless indicated 
otherwise in a credit line to the material. If material is not included in the article's Creative Commons licence and your intended use is not permitted by statutory regulation or exceeds the permitted use, you will need to obtain permission directly from the copyright holder. To view a copy of this licence, visit http://creativecommons.org/licenses/by/4.0/.

\section{References}

Adra N, Abonour R, Althouse SK, Albany C, Hanna NH, Einhorn LH (2017) High-dose chemotherapy and autologous peripheral-blood stem-cell transplantation for relapsed metastatic germ cell tumors: the Indiana University experience. J Clin Oncol 35:1096-1102

Albers P, Albrecht W, Algaba F, Bokemeyer C, Cohn-Cedermark G, Fizazi K et al (2015) Guidelines on testicular cancer: 2015 update. Eur Urol 68:1054-1068

Beyer J, Albers P, Altena R, Aparicio J, Bokemeyer C, Busch J et al (2013) Maintaining success, reducing treatment burden, focussing on survivorship: highlights from the third European Consensus Conference on Diagnosis and Treatment of Germ Cell Cancer. Ann Oncol 24:878-888

Beyer J, Collette L, Daugaard G, de Wit R, Tyrakin A, Albany C et al. (2020) Prognostic factors in advanced seminoma: an analysis from the IGCCCG update consortium. J Clin Oncol 38(6) (Suppl.): abstract 386.

Cathomas R, Hartmann M, Krege S, Souchon R, Lorch A, Mayer F et al (2011) Interdisciplinary evidence-based recommendations for the follow-up of testicular germ cell cancer patients. Onkologie 34:59-64

Daugaard G, Skoneczna I, Aass N, De Wit R, De Santis M, Dumez H et al (2011) A randomized phase III study comparing standard dose BEP with sequential high-dose cisplatin, etoposide, and ifosfamide (VIP) plus stem-cell support in males with poor-prognosis germ-cell cancer. An intergroup study of EORTC, GTCSG, and Grupo Germinal (EORTC 30974). Ann Oncol 22:1054-1061

De Wit R, Skoneczna I, Daugaard G, De Santis M, Garin A, Aass N et al (2012) Randomized phase III study comparing paclitaxelbleomycin, etoposide, and cisplatin to standard BEP in intermediate prognosis germ cell-cancer. Intergroup Study EORTC 30983. J Clin Oncol 30:792-799

Fankhauser CD, Sander S, Roth L, Beyer J, Hermanns T (2018) Improved survival in metastatic germ-cell cancer. Ann Oncol 29:347-351

Fenner M, Oing C, Dieing A, Gauler T, Oechsle K, Lorch A et al (2019) Everolimus in patients with multiply relapsed or cisplatin refractory germ cell tumors: results of a phase II, single-arm, open-label multicenter trial (RADIT) of the German Testicular Cancer Study Group. J Cancer Res Clin Oncol 145:717-723

Fizazi K, Pagliaro L, Laplanche A, Fléchon A, Mardiak J, Geoffrois L et al (2014) Personalised chemotherapy based on tumour marker decline in poor prognosis germ-cell tumours (GETUG 13): a phase 3, multicentre, randomised trial. Lancet Oncol 15:1442-1450

Gerl A, Clemm C, Schmeller N, Hartenstein R, Lamerz R, Wilmanns W (1996) Advances in the management of metastatic non-seminomatous germ cell tumours during the cisplatin era: a singleinstitution experience. Br J Cancer 74:1280-1285

Gillessen S, Collette G, Daugaard G, de Wit R, Tryakin A, Albany C et al (2019) Redefining the IGCCCG classification in advanced non-seminoma. Ann Oncol 30(Suppl. 5):v357-358

Hanna NH, Einhorn LH (2014) Testicular cancer-discoveries and updates. New Engl J Med 371:2005-2016

Honecker F, Aparicio J, Berney D, Beyer J, Bokemeyer C, Cathomas $\mathrm{R}$ et al (2018) ESMO Consensus Conference on testicular germ cell cancer: diagnosis, treatment and follow-up. Ann Oncol 29:1658-1686
International Prognostic Factors Study Group, Lorch A, Beyer J, Bascoul-Mollevi C, Kramar A, Einhorn LH et al (2010) Prognostic factors in patients with metastatic germ cell tumors who experienced treatment failure with cisplatin-based first line chemotherapy. J Clin Oncol 28:4906-4911

Kondagunta GV, Bacik J, Donadio A, Bajorin D, Marion S, Sheinfeld $\mathrm{J}$ et al (2005) Combination of paclitaxel, ifosfamide, and cisplatin is an effective second-line therapy for patients with relapsed testicular germ cell tumors. J Clin Oncol 23:6549-6555

Lorch A, Kleinhans A, Kramar A, Kollmannsberger CK, Hartmann JT, Bokemeyer C et al (2012) Sequential versus single highdose chemotherapy in relapsed or refractory germ cell tumors: long-term results of a prospective randomized trial. J Clin Oncol 30:800-805

Motzer RJ, Nichols CL, Margolin KA, Bacik J, Richardson PG, Vogelzang NJ et al (2007) Phase III randomized trial of conventionaldose chemotherapy with or without high-dose chemotherapy and autologous hematopoietic stem-cell rescue as first-line treatment for patients with poor-prognosis metastatic germ cell tumors. J Clin Oncol 25:247-256

Necchi A, Mariani L, Di Nicola M, Lo Vullo S, Nicolai N, Giannatempo $\mathrm{P}$ et al (2015) High-dose sequential chemotherapy (HDS) versus PEB chemotherapy as first-line treatment of patients with poor prognosis germ-cell tumors: mature results of an Italian randomized phase II study. Ann Oncol 26:167-172

Nichols CR, Catalano PJ, Crawford ED, Vogelzang NJ, Einhorn LH, Loehrer PJ (1998) Randomized comparison of cisplatin and etoposide and either bleomycin or ifosfamide in treatment of advanced disseminated germ cell tumors: an Eastern Cooperative Oncology Group, Southwest Oncology Group, and Cancer and Leukemia Group B Study. J Clin Oncol 16:1287-1293

Raggi D, Mariani L, Giannatempo P, Lo Vullo S, Giardiello D, Nicolai $\mathrm{N}$ et al (2015) Prognostic reclassification of patients with intermediate-risk metastatic germ cell tumors: Implications for clinical practice, trial design, and molecular interrogation. Urol Oncol 33:332.e19-24

Robert Koch-Institute (2019) Zentrum für Krebsregisterdaten. https ://www.krebsdaten.de/Krebs/DE/Content/Publikationen/Krebs _in_Deutschland/kid_2019/kid_2019_c62_hoden.pdf. Access on 17 July 2020

Seidel C, Daugaard G, Tryakin A, Necchi A, Cohn Cedermark G, Ståhl $\mathrm{O}$ et al (2018) Intermediate prognosis in metastatic germ cell tumours-outcome and prognostic factors. Eur J Cancer 94:16-25

Seidel C, Daugaard G, Tryakin T, Necchi A, Cohn-Cedermark G, Ståhl O et al (2019) The prognostic impact of different tumor marker levels in nonseminomatous germ cell tumor patients with intermediate prognosis: a registry of the International Global Germ Cell Tumor Collaborative Group (G3). Urol Oncol 37:809.e19-809. e25

The International Germ Cell Cancer Collaborative Group (1997) International Germ Cell Consensus Classification: a prognostic factor-based staging system for metastatic germ cell cancers. J Clin Oncol 15:594-603

van Dijk MR, Steyerberg EW, Habbema JD (2006) Survival of nonseminomatous germ cell cancer patients according to the IGCCG classification: an update based on meta-analysis. Eur J Cancer 42:820-826

Williams SD, Birch R, Einhorn LH, Irwin L, Greco FA, Loehrer PJ (1987) Treatment of disseminated germ-cell tumors with cisplatin, bleomycin, and either vinblastine or etoposide. N Engl J Med 316:435-1440

Publisher's Note Springer Nature remains neutral with regard to jurisdictional claims in published maps and institutional affiliations. 